\title{
Preface to the Special Section: Infinity in Philosophy and Mathematics
}

\author{
Sakaé Fuchino*
}

The symposium "Infinity in Philosophy and Mathematics" took place during the annual meeting of the Japan Association for Philosophy of Science held at Senshu University Tokyo on June 12, 2010. The symposium included keynote lectures of Professors Juliet Floyd and Akihito Kanamori of Boston University, and a panel discussion with Professors Floyd and Kanamori as well as Professor Yasuyuki Kawahigashi of Tokyo University and Professor Kazuhisa Todayama of Nagoya University.

The present special section in the Annals of the Japan Association for Philosophy of Science is created in connection with this symposium. The section contains articles by Akihiro Kanamori, John P. Burgess, Toshiyasu Arai and Sakaé Fuchino. "The Mathematical Infinite as a Matter of Method" by Akihiro Kanamori is based on his keynote lecture at the symposium and addresses the historical emergence of the mathematical infinite and the question how we are to take the infinite in and out of mathematics. John P. Burges discusses in his "Axioms of Infinity as the Starting Point for Rigorous Mathematics" the mathematical infinity from the point of view of axiomatization. "A Sneak Preview of Proof Theory of Ordinals" by Toshiyasu Arai reviews the ordinal analysis in proof theory and previews some results in his project on "proof theory for theories of ordinals". The short article "The Set-theoretic multiverse as a mathematical plenitudinous Platonism viewpoint" discusses possible forms of mathematical Platonism in wake of resent developments in set theory.

Of course, "the infinity is a large topic" as Professor Kanamori put it in his article. I hope very much that the discussions in the symposium and the articles collected here will serve as a beginning of further fruitful colloquy on this large topic.

\section{* Guest editor.}

Kobe University, Graduate School of System Informatics

Rokko-dai 1-1, Nada, Kobe 657-8501

E-mail: fuchino@diamond.kobe-u.ac.jp

I would like to thank Professors Takashi Iida, Hiroshi Kaneko and Makoto Kikuchi for their cooperation and assistance for the organization of the symposium. Many additional thanks are also due to Professor Kikuchi for his valuable help in the editing of this special section. 